\title{
Spiral graphone and one-sided fluorographene nanoribbons
}

\author{
M. Neek-Amal, ${ }^{1,2, *}$ J. Beheshtian, ${ }^{2}$ F. Shayeganfar, ${ }^{3}$ S. K. Singh, ${ }^{1}$ J. H. Los, ${ }^{4}$ and F. M. Peeters ${ }^{1}$ \\ ${ }^{1}$ Departement Fysica, Universiteit Antwerpen, Groenenborgerlaan 171, B-2020 Antwerpen, Belgium \\ ${ }^{2}$ Department of Physics, Shahid Rajaee Teacher Training University, Lavizan, Tehran 16785-136, Iran \\ ${ }^{3}$ Département de Génie Physique et Regroupement Québécois sur les Matériaux de Pointe (RQMP), École Polytechnique de Montréal, C.P., \\ succursale Centre-Ville, Montréal, Québec, Canada H3C $3 A 7$ \\ ${ }^{4}$ Institute of Physical Chemistry and Center for Computational Sciences, Johannes Gutenberg University Mainz, \\ Staudinger Weg 9, D-55128 Mainz, Germany
}

(Received 20 December 2012; revised manuscript received 30 January 2013; published 27 February 2013)

\begin{abstract}
The instability of a free-standing one-sided hydrogenated/fluorinated graphene nanoribbon, i.e., graphone/fluorographene, is studied using ab initio, semiempirical, and large-scale molecular dynamics simulations. Free-standing semi-infinite armchairlike hydrogenated/fluorinated graphene (AC-GH/AC-GF) and boatlike hydrogenated/fluorinated graphene (B-GH/B-GF) (nanoribbons which are periodic along the zigzag direction) are unstable and spontaneously transform into spiral structures. We find that rolled, spiral B-GH and B-GF are energetically more favorable than spiral AC-GH and AC-GF which is opposite to the double-sided flat hydrogenated/fluorinated graphene, i.e., graphane/fluorographene. We found that the packed, spiral structures exhibit an unexpected localized highest occupied molecular orbital and lowest occupied molecular orbital at the edges with increasing energy gap during rolling. These rolled hydrocarbon structures are stable beyond room temperature up to at least $T=1000 \mathrm{~K}$ within our simulation time of $1 \mathrm{~ns}$.
\end{abstract}

DOI: 10.1103/PhysRevB.87.075448

PACS number(s): 73.23.-b, 73.21.La, 72.15.Qm, 71.27.+a

\section{INTRODUCTION}

The discovery of graphene ${ }^{1}$ has been a driving force for the scientific community to synthesize and to characterize new materials with similar morphologies due to their unique properties. ${ }^{2-5}$ Fully double-sided hydrogenated graphene, i.e., graphane, and double-sided fluorographene are quasi-twodimensional lattices of carbon $(\mathrm{C})$ atoms ordered into a buckled honeycomb sublattice, where each carbon atom is covalently bonded to hydrogen $(\mathrm{H})$ or fluor $(\mathrm{F})$, respectively, in an alternating chairlike arrangement. ${ }^{6,7}$ The chemisorption of hydrogen and fluor atoms results in an important reconstruction of the chemical bonds and angles of the underlying honeycomb lattice. ${ }^{8}$ This transition from $s p^{2}$ to $s p^{3}$ hybridization turns the graphitic $\mathrm{C}-\mathrm{C}$ bonds into single bonds by the formation of additional single $\mathrm{C}-\mathrm{H}$ and $\mathrm{C}-\mathrm{F}$ bonds, which change locally the planar shape of graphene into an out-of-plane, angstrom-scale buckled geometry. ${ }^{9}$

Experimentally, it has been shown that graphane can be obtained reversibly starting from a pure graphene layer in the presence of atomic hydrogen. ${ }^{10}$ It has become an interesting material due to its potential applications in nanoelectronics. ${ }^{11-16}$ On the other hand, the experimentally obtained single-layer fluorographene exhibits a strong insulating behavior with a room-temperature resistance larger than $1 \mathrm{~T} \Omega$, and a high-temperature stability up to $400{ }^{\circ} \mathrm{C}$. $^{7}$

Both in experiments ${ }^{10}$ and in ab initio calculations, flat single-sided hydrogenated graphene, i.e., graphone $(\mathrm{GH})$, was found to be unstable, ${ }^{17,18}$ which was also demonstrated by phonon band-structure calculations. Supporting graphene on a substrate and hydrogenating was demonstrated to produce $\mathrm{GH}$ in a recent experiment. ${ }^{19} \mathrm{Ab}$ initio calculations showed that armchair (AC) graphane is more stable than boatlike (B) graphane, while B-GH was found to be more stable than AC-GH. ${ }^{20}$ Ab initio calculations also showed that $\mathrm{GH}$ exhibits magnetism due to the localized electrons on the carbon atoms without hydrogens, in contrast to nonmagnetic graphene and graphane. ${ }^{21,22}$

By using both $a b$ initio calculations and elasticity theory, Kudin et al. found that while linear elasticity theory can be used to study the stiffness and flexural rigidity moduli of carbon and boron-nitride nanoshells, one-sided fluorinated carbon ribbons exhibit a strong tendency to shell formation resulting in very-small-diameter tubes with $(4,4)$ and $(3,3)$ indexes and properties that deviate from linear elasticity theory. ${ }^{23}$ Such a strong tendency to bending is also observed for $\mathrm{Si}$ and bilayer SiGe nanofilms, due to reconstructions in the surface layer ${ }^{24}$ associated with dimer formation. Although the possible deformation of the edge of graphene (see Ref. 25 and references therein) and the synthesis of carbon nanotubes via one-sided hydrogenation or fluorination of supported carbon films have been investigated theoretically, ${ }^{26}$ the geometrical structure of long (referring to the distance between the free edges) nanoribbons of graphone and their corresponding stability and electronic properties at room temperature have not been studied so far. Here we show that such long nanoribbons of graphone and fluorinated graphene (GH/GF) do not form carbon nanotubes, as in the case of relatively short samples with specific sizes, ${ }^{26}$ but, instead, spontaneously form rolled, spiral structures with interesting localization of frontier molecular orbitals. The one-sided absorption and corresponding $s p^{3}$ hybridization is the main driving force for this transformation, and the final spiral structure is significantly more stable than the corresponding flat structures. We investigate various electronic and geometrical properties of these spiral structures, which we name spiral graphone (fluorinated graphene).

There have been several recent experimental and theoretical studies on the formation of carbon nanotubes using a graphene sheet and inverse processing. ${ }^{27-29}$ Much experimental evidence point towards the possibility of the production of the spiral carbon nanostructures proposed here. In a recent review 
paper, Vilatela and Eder $^{30}$ reviewed related experimental studies, compared the potentials and characteristics of nanocarbon composites (such as various rolled sheets), and discussed key challenges for the use of the new carbon nanostructures. Furthermore, similar rolled structures for $\mathrm{Ni}_{3} \mathrm{Si}_{2} \mathrm{O}_{5}(\mathrm{OH})_{4}$ were synthesized and analyzed using $\mathrm{X}$-ray diffraction (XRD) and transmission electron microscopy (TEM). ${ }^{31}$ Shen et al. ${ }^{32}$ reported recently the synthesis of self-assembled kinked $\mathrm{In}_{2} \mathrm{O}_{3}$ nanospirals and multikinked nanowires using a tube-in-tube laser ablation chemical vapor deposition (CVD) method with gold nanoparticles as catalysts.

Apart from being more stable than flat structures, we also show that spiral B-GH and B-GF are more stable than spiral AC-GH and AC-GF, respectively. The spiral structures are closely packed and stable even at $1000 \mathrm{~K}$ and are candidates for a separate class of carbon nanostructures. Since the deformations for the GFs and the GHs are very similar, we will report here mainly the results for the GHs. After rolling, the highest occupied molecular orbital (HOMO) and the lowest unoccupied molecular orbital (LUMO) are separated and appear to be localized at opposite (free) ends, making these two ends chemically more active ${ }^{33}$ while before and during rolling they are localized at both ends simultaneously. We found that the rolling process makes the systems strongly polarized and more insulating.

\section{METHODS AND MODELS}

We performed $a b$ initio calculations using GAUSSIAN (G09), ${ }^{34}$ which is an electronic structure package that uses a basis set of Gaussian-type orbitals. In the ab initio calculations for the exchange and correlation (XC) functional, the hybrid functional B3LYP is adopted in G09. The self-consistency loop was iterated until the change in the total energy is less than $10^{-7} \mathrm{eV}$, and the geometries were considered relaxed once the force on each atom is less than $50 \mathrm{meV} / \AA$. Using the $6-31 G^{*}$ basis set in $\mathrm{G} 09$, we expect that our calculation is capable of providing a reliable description of the electronic properties of the different systems. In the case of AC-GH, the A-sublattice sites of graphene were covered with hydrogens, while the B-GH model was obtained by hydrogenating all carbon atoms involved in horizontal $\mathrm{C}-\mathrm{C}$ bonds along the $\mathrm{AC}$ direction. Hence the number of carbon atoms is twice as large as the number of hydrogen atoms. The simulated samples [using the density-functional theory (DFT) method] have typically more than 500 atoms.

For the larger samples, we performed semiempirical calculations (PM6 level of calculation in G09) as well as classical molecular dynamics (MD) simulations using the LAMMPS package. $^{35}$

For the classical MD simulations, the modified second generation of Brenner's bond-order potential, i.e., the adaptive intermolecular reactive bond order (AIREBO), ${ }^{36}$ and the reactive force field (ReaxFF) ${ }^{37}$ potential were employed to simulate the GHs and GFs, respectively. These simulations were performed using periodic boundary conditions along the lateral side (perpendicular to the rolling direction, i.e., armchair) in the canonical NVT ensemble with a Nosé-Hoover thermostat for temperature control and a time step of $0.1 \mathrm{fs}$. The simulation box size along the rolling direction was taken equal to twice the system size (in its flat geometry) in that direction.

AIREBO consists of two parts, namely, the reactive bond order potential $(\mathrm{REBO})^{38}$ for the short-range interactions ( $<2 \AA$ ), but with a modified, four-body, bond-order contribution for the torsion in various hydrocarbon configurations, and the van der Waals (vdW) term for long-range interactions within distances $2<r<10 \AA$, which is similar to the standard Lennard-Jones potential, but with an environment-dependent (adaptive) suppression of the (too) strong $1 / r^{12}$ repulsion. Its total energy reads

$$
E_{\mathrm{AIREBO}}=E_{\mathrm{REBO}}^{\prime}+E_{v d W},
$$

where the prime in $E_{\mathrm{REBO}}^{\prime}$ is added to indicate the modification in the torsion term. In our MD simulations, we checked the effect of the vdW term (by turning it on/off) to ensure that when GH is rolled up, the vdW term does not prevent possible bonding between two close-edge atoms. The simulations where we included the vdW term are labeled with an asterisk. We found that including the vdW term lowers the energy of spiral $\mathrm{GH}^{*}$ by only $0.04 \mathrm{eV} /$ atom with respect to $\mathrm{GH}$.

In ReaxFF (for simulating B/AC-GFs), the atomic interactions are described by a reactive force field potential. ${ }^{37}$ ReaxFF is a general bond-order-dependent potential that provides an accurate description of the bond breaking and bond formation. Recent simulations on a number of hydrocarbon-oxygen systems showed that ReaxFF reliably provides energies, transition states, reaction pathways, and reactivity in reasonable agreement with $a b$ initio calculations and experiments.

For our MD simulations, we considered rectangular GH nanoribbons containing different number of carbon atoms, namely, $N_{C}=4800$ and 5760 . For the GFs, we took $N_{C}=$ 5600. For the semiempirical calculations at the PM6 level, we took $N_{C}=1500$.

\section{RESULTS AND DISCUSSION}

\section{A. Infinite one-sided hydrogenated graphene}

Figure 1 shows two side views of relaxed infinite (with periodic boundary condition applied in both directions of the plane) B-GH [Fig. 1(a)] and AC-GH [Fig. 1(b)] at $T=10 \mathrm{~K}$ using the $\mathrm{MD}$ method. In the $\mathrm{AC}-\mathrm{GH}$ configuration, the hydrogens are relatively far from each other and the $\mathrm{C}-\mathrm{H}$ bonds are mutually parallel and perpendicular to the $z=0$ plane due to symmetry. In the B-GH configuration, the $\mathrm{C}-\mathrm{H}$ bonds are attached to the nearest-neighbor $\mathrm{C}-\mathrm{C}$ bond, which induces a corrugation in the hydrogen layer with $\mathrm{C}-\mathrm{H}$ bonds that are not fully perpendicular to the $z=0$ plane, mainly because of the $s p^{3}$ hybridization but also due to the stronger repulsion between the relatively close hydrogen atoms. Very similar configurations were obtained for GF, which are therefore not shown. It is important to note that such flat GHs (and one-sided GFs) are energetically unfavorable. ${ }^{17}$ In this study, we report on the structural transformations of these systems into stable, nonflat structures. Notice that the rolling direction in this study is along the armchair direction. However, the rolling effect is also found along the zigzag direction, as shown in one of the movies in the Supplemental Material. ${ }^{39}$ 

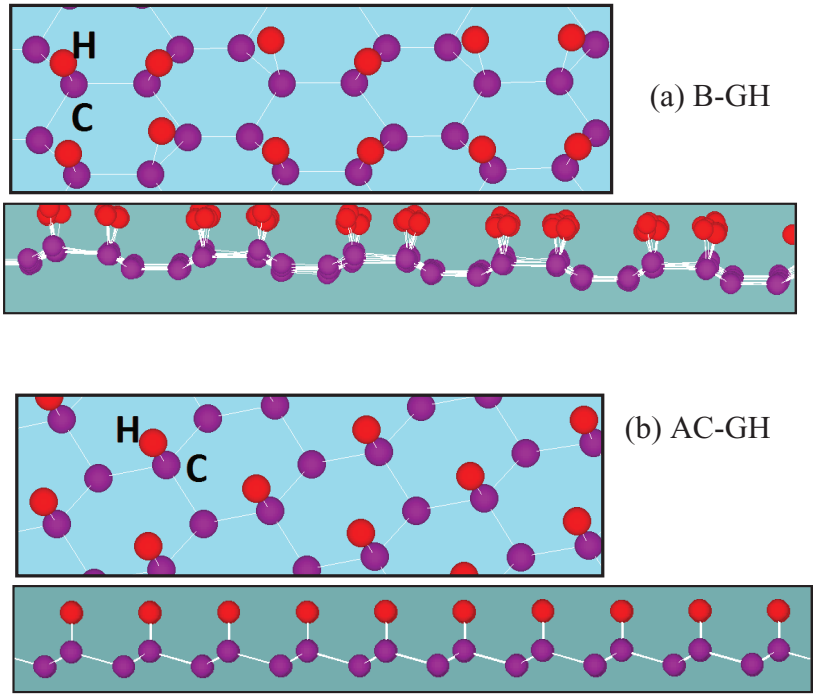

FIG. 1. (Color online) Top and side views (along armchair direction) of a small portion of simulated graphones which were relaxed at $T=10 \mathrm{~K}$ using molecular dynamics simulation. (a) Boatlike graphone and (b) armchair graphone.

\section{B. Ab initio results: Graphone}

In Fig. 2(a), we show the optimized configuration of B-GH using $a b$ initio calculations with B3LYP/6-31G* (neglecting spin-polarization effects) and 504 atoms. Notice that the initial nonrelaxed configuration was a flat sheet. In Fig. 2(b), an example of results from our semiempirical calculation using PM6 is shown.

Figure 3(a) shows the electrostatic surface potential (ESP) of $\mathrm{B}-\mathrm{GH}$ before the completion of the rolling process. The corresponding HOMO and LUMO are shown in Figs. 3(b) and 3(c), respectively. During rolling, both HOMO and LUMO are localized at both ends simultaneously, which is a consequence of the equivalent geometry. The ESP of completely rolled B-GH is shown in Fig. 4(a). The ESP indicates that the spiral structure has ionic characteristics, with the red (blue) color indicating positively (negatively) charged regions, which stabilizes the spiral geometry even more.

Since hydrogen has a smaller electron affinity than carbon, the ESP at the side covered by $\mathrm{H}$ atoms is higher, as indicated

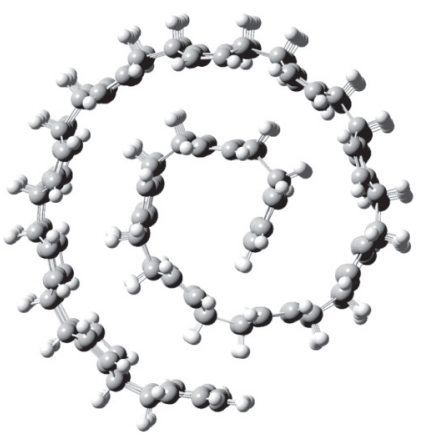

(a)

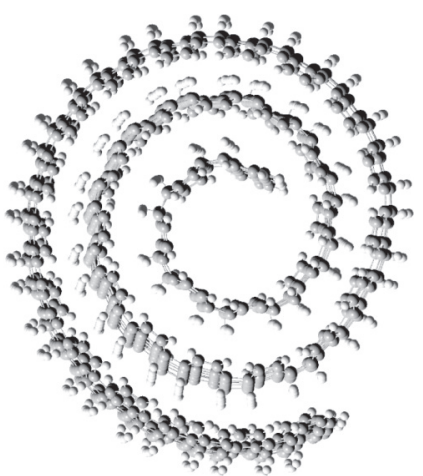

(b)
FIG. 2. (a) The spiral B-GH using DFT calculations for 504 atoms. (b) The spiral B-GH using semiempirical calculations (PM6) for 1320 atoms.

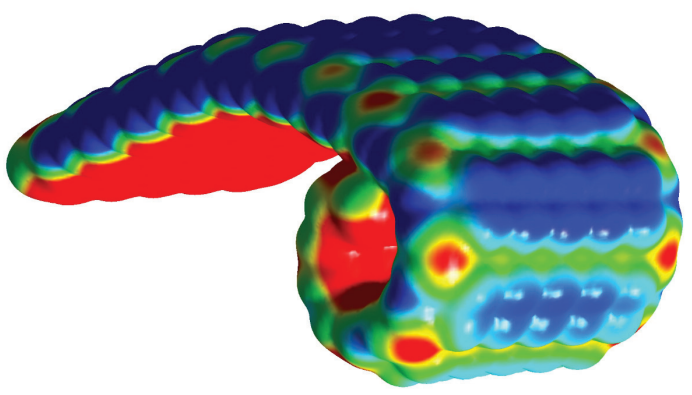

(a)

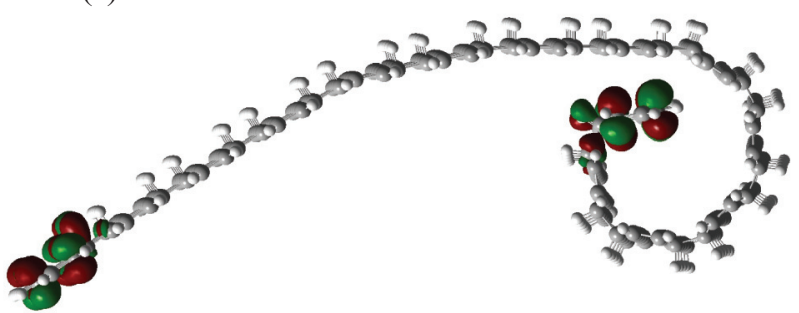

(b)

$\mathrm{HOMO}$

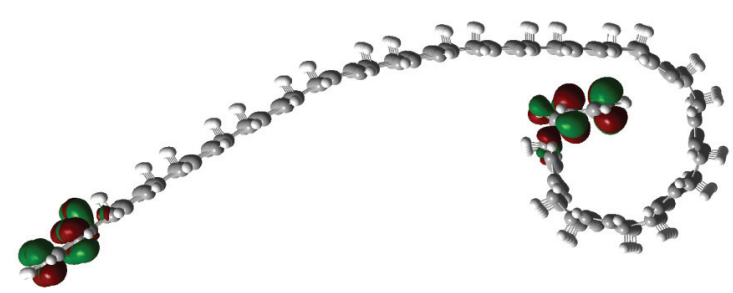

(c) LUMO

FIG. 3. (Color online) (a) Electrostatic potential and the (b) HOMO and (c) LUMO for B-GH during rolling.

with blue spots in Figs. 3(a) and 4(a), while the bare side has a lower (higher) ESP, indicated with red color. When the sheet starts to roll up, the inner edge that is located between the two bare sides experiences lower ESP, contrary to the outer edge. Therefore, the region around the inner edge is the repulsive region for both $\mathrm{GH}$ (electron-electron interaction). The negative charge of the electron results in an increment of the energy of the electrons close to the inner edge. On the other hand, during the rolling process, the outer edge approaches the $\mathrm{H}$-covered side with high EPS and therefore the energy of the electrons living close to this edge decreases. The resulting intrinsic potential gradient propagates along the sheet and an electron transfer occurs from the inner part towards the outer end. One can equivalently argue that the electrons have lower energy in the vicinity of positively charged hydrogen atoms as compared to the situation where they are surrounded by the negatively charged carbons. Thus, they leave the inner edge, leading to an electron accumulation at the outer edge. This induces a polarization across the two edges. Note that the sheet is simultaneously polarized across the sheet, in the direction normal to its plane pointing towards the center.

Surprisingly, after complete rolling, HOMO and LUMO are separated and both are localized in only one end, as can be seen from Figs. 4(b) and 4(c), whereas before complete rolling, they were localized at both ends simultaneously as already mentioned before [see Figs. 3(b) and 3(c)]. Because of 


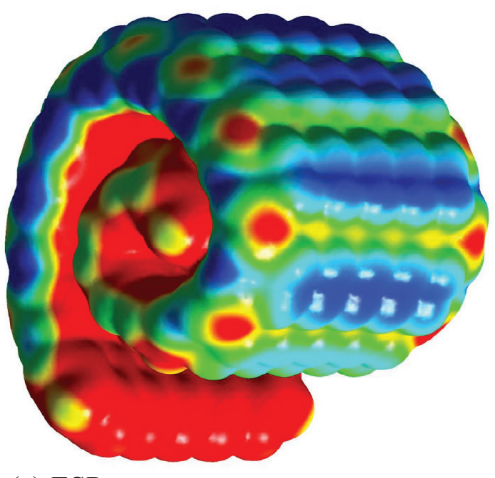

(a) ESP

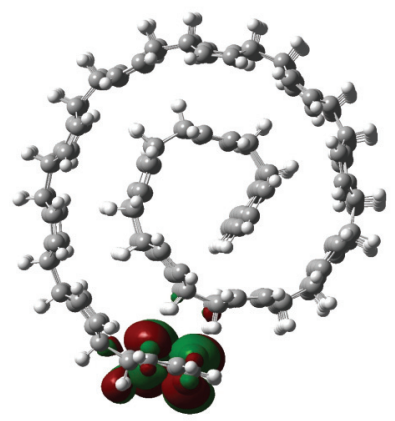

(b) HOMO

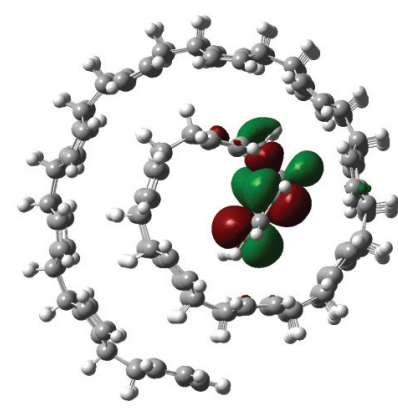

(c) LUMO
FIG. 4. (Color online) (a) Electrostatic potential around spiral graphone and the (b) HOMO and (c) LUMO of the system.

the different ESP experienced at the two edges, the increment of the energy of the LUMO is more than that of the HOMO, which for spiral $\mathrm{GH}$ results in a broadening of the energy gap between them (Fig. 5) towards about $0.3 \mathrm{eV}$. As shown in Fig. 5, the energy gap increases almost linearly during the evolution to a spiral, indicating that spiral $\mathrm{GH}$ is more insulating than flat graphone.

Because of the difference in ESP at the two edges, the increment of the energy of the LUMO is larger than that of the HOMO, which results in a widening of the energy gap from $0.1 \mathrm{eV}$ for the flat sheet to $0.3 \mathrm{eV}$ for the spiral structure. This is shown more explicitly in Fig. 5, which reveals an almost linear increase of the gap during the evolution to a spiral.

\section{C. $A b$ initio results: One-sided fluorinated graphene}

Very similar results are found for GFs. Figure 6(a) shows the spiral GFs obtained using B3LYP/6-31G*. The spiral GF formed using the PM6 method is shown in Fig. 6(b). These configurations are minimum-energy configurations of B-GF. Similarly, as for $\mathrm{GH}$, the rolling is due to the one-sided, partial (here $50 \%$ ) coverage with $\mathrm{F}$ atoms and corresponding $s p^{3}$ hybridization. The rolling process starts from free boundaries where there is no resistance against any torque. The corresponding ESP, HOMO and LUMO are shown in Figs. 7(a)-7(c), respectively.

\section{Classical molecular dynamics simulation results: Large-size flakes}

Using classical simulations, we first investigated the possibility to create hydrogenated carbon nanotubes (CNTs) from one-sided hydrogenated AC-GH nanoribbons, with periodic

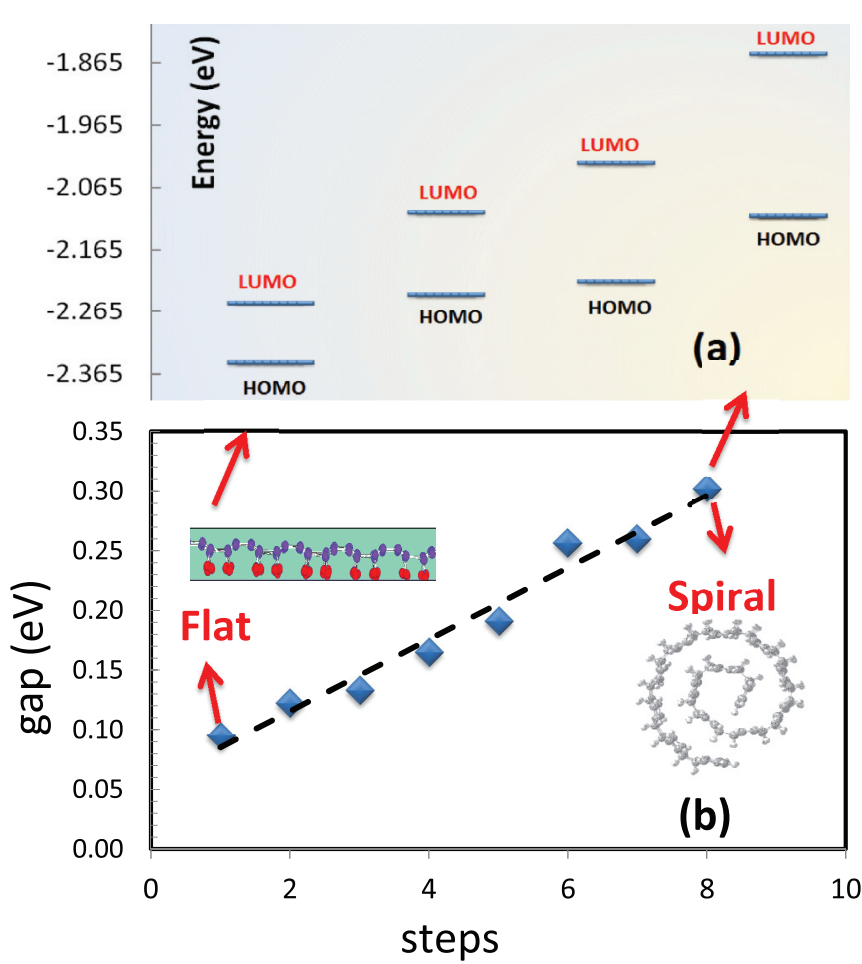

FIG. 5. (Color online) (a) HOMO-LUMO energy diagram for four different instances of the rolling process and (b) the variation of the energy gap during rolling (for eight steps) for the B-GH shown in Fig. 2 (left)

boundary condition along the zigzag edge, having a specific length equal to $L=13.4 \AA$ between the two free ends (in the flat geometry). After relaxing the system at $10 \mathrm{~K}$, we found that a $(5,5)$ CNT is always formed, independent of the dimension of the box in the periodic direction (see movie in Supplemental Material). ${ }^{39}$ The used length for B-GH, i.e., $L=13.4 \AA$, agrees with the perimeter of a one-sided hydrogenated $(5,5)$ CNT, i.e., $5 \sqrt{3} a_{0}$ with $a_{0} \cong 1.5 \AA$. The movie in Ref. 39 shows the time evolution for the rolling of an $\mathrm{AC}-\mathrm{GH}$, which eventually forms a $(5,5)$ hydrogenated CNT. This is consistent with previous results on the formation of CNTs from partially hydrogenated graphene. ${ }^{26}$ Note, however, that the formation of these nanotubes is constrained by the choice of the length of

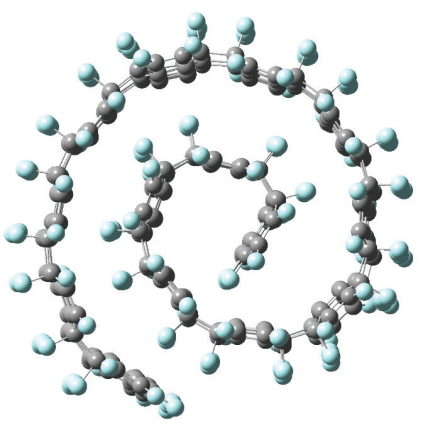

(a)

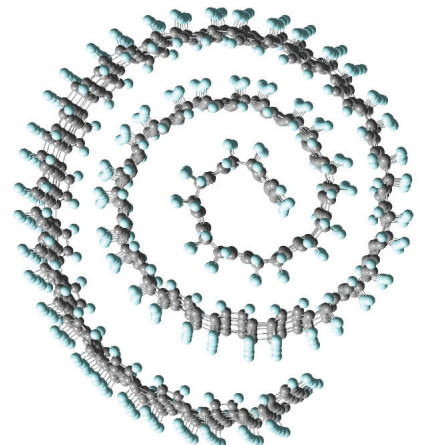

(b)
FIG. 6. (Color online) (a) Spiral B-GF from DFT calculations for 504 atoms. (b) Spiral B-GF from semiempirical calculations (PM6) for 1428 atoms. 


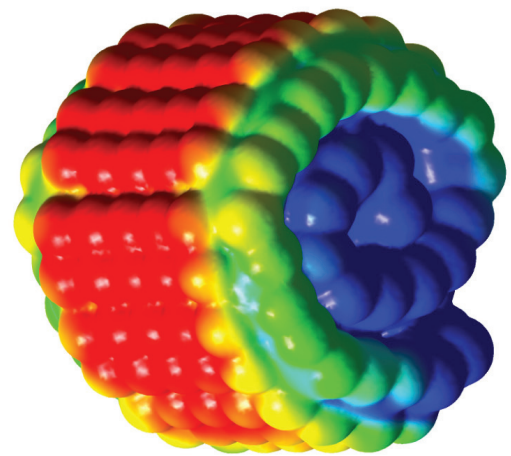

(a)

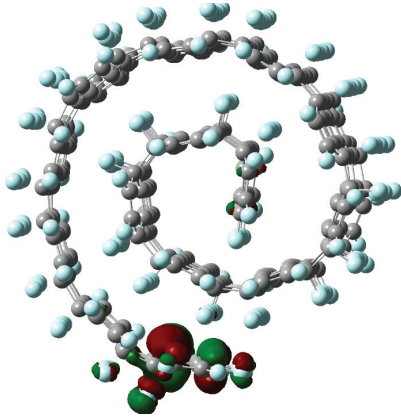

(b) $\mathrm{HOMO}$

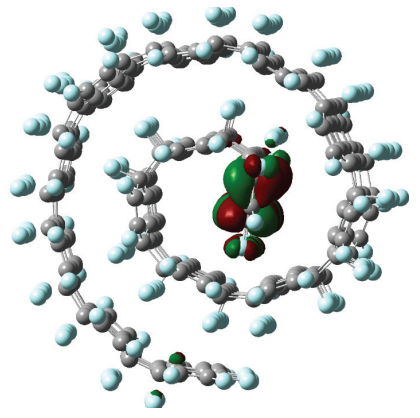

(c) LUMO
FIG. 7. (Color online) (a) ESP, (b) HOMO, and (c) LUMO for the B-GF system shown in Fig. 6 (left).

the ribbon, which has to be relatively small and should match with the size of a $(n, n)$ tube.

In the remainder of this section, we show the rolling effect for much longer nanoribbons, not leading to tube formation, using classical MD simulation. We used graphone nanoribbons with free zigzag edges and periodic boundary conditions in the direction parallel to these edges (lateral side; see Fig. 1) and found that the final, minimal-energy configuration of both $\mathrm{GH}$ and GF is a spiral structure similar to what we found with DFT. Figures 8(a) and 8(b) show two snapshots of the rolled $\mathrm{B}-\mathrm{GH}$ which are free at the two longitudinal ends (here set to be zigzag edges). Similar results are found for AC-GHs; see Figs. 8(c) and 8(d). As expected, the corrugations in B-GH are larger than in $\mathrm{AC}-\mathrm{GH}$. Thus, unrolling $\mathrm{B}-\mathrm{GH}$ costs much more energy than AC-GH.

Figure 9 shows the variation of the total energy per atom as a function of the rolling process time for AC-GH, B-GH,

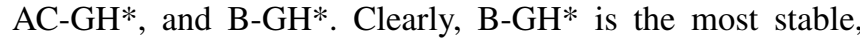
having the lowest energy. The energy gap between AC-GH $\left(\mathrm{AC}-\mathrm{GH}^{*}\right)$ and $\mathrm{B}-\mathrm{GH}\left(\mathrm{B}-\mathrm{GH}^{*}\right)$ is about $0.16 \mathrm{eV} /$ atom. Furthermore, it is seen that including the vdW energy term lowers the energy slightly, by $\sim 0.04 \mathrm{eV} /$ atom, due to the attractive van der Waals interaction. Notice that the ionic characteristic of the rolled structure cannot be reproduced by these classical simulations with AIREBO. Nonetheless, the classically found spiral structures are quite stable, which confirms that the main driving force for the rolling is the one-sided absorption and corresponding $s p^{3}$ hybridization and not the ionic characteristics found in the DFT calculations.

In Fig. 10, rolled configurations of a system consisting of two connected GH parts that are hydrogenated at opposite sides are shown. Hydrogens at $x<0(x>0)$ are bonded to the upper (lower) side of the sheet. Notice that the AC-GH and B-GH form different patterns of rolled $\mathrm{GH}$, with an extra rolled part in the middle. This hints at the interesting possibility to engineer the rolled shape of GH carefully by selectively hydrogenating parts of the initial graphene sheet.

As an additional, final result of this section, we present the rolling behavior of a system with one side covered by hydrogen and the other side covered by fluor, which we called "GFH." Recently, by using ab initio calculations, a negative formation energy was found for this system. ${ }^{40}$ In Fig. 11, side views are shown for the rolled structure of AC-GF [Fig. 11(a)], AC-GH
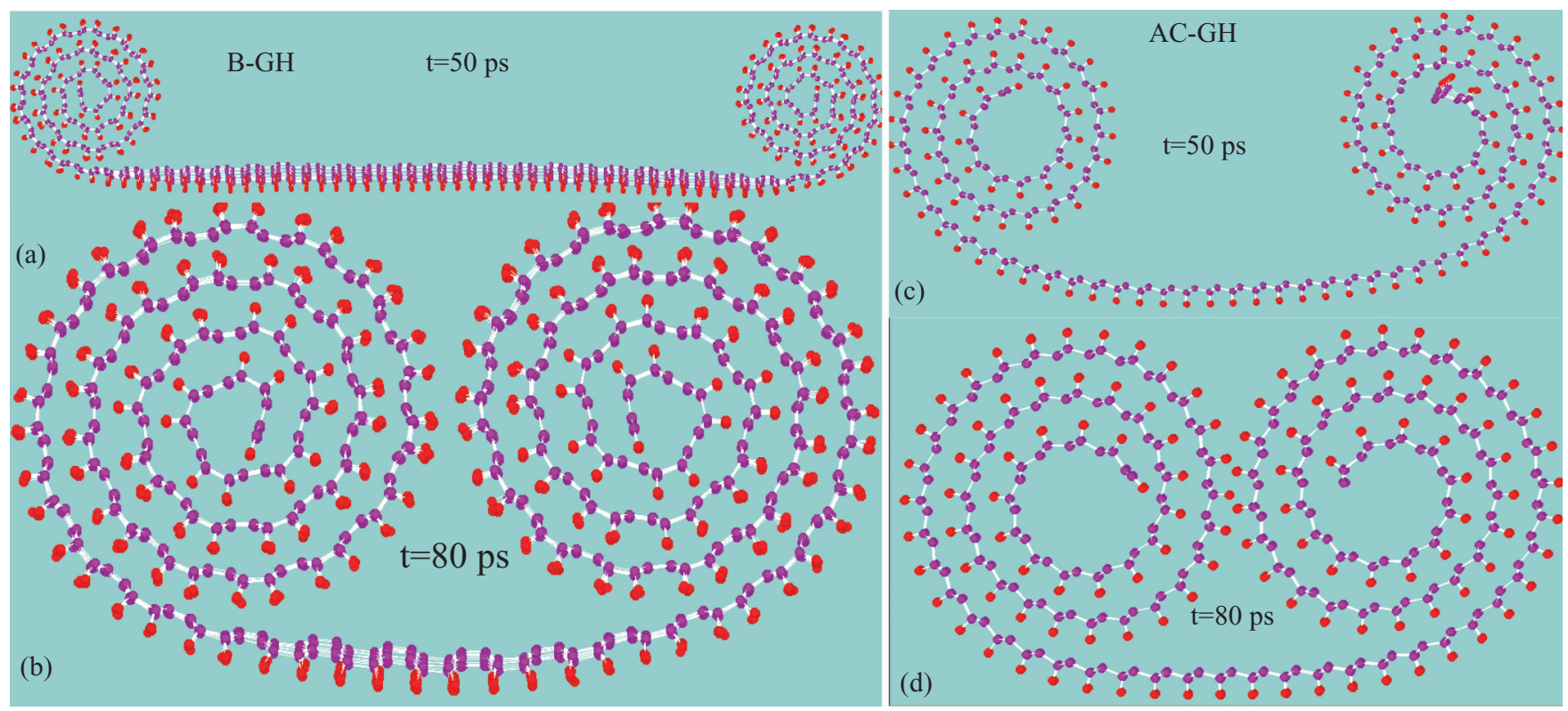

FIG. 8. (Color online) The rolled boatlike (left) and armchairlike (right) graphone nanoribbons after (a),(c) $t=50 \mathrm{ps}$ and (b),(d) $t=80$ ps. These systems are stable up to at least $1000 \mathrm{~K}$. 


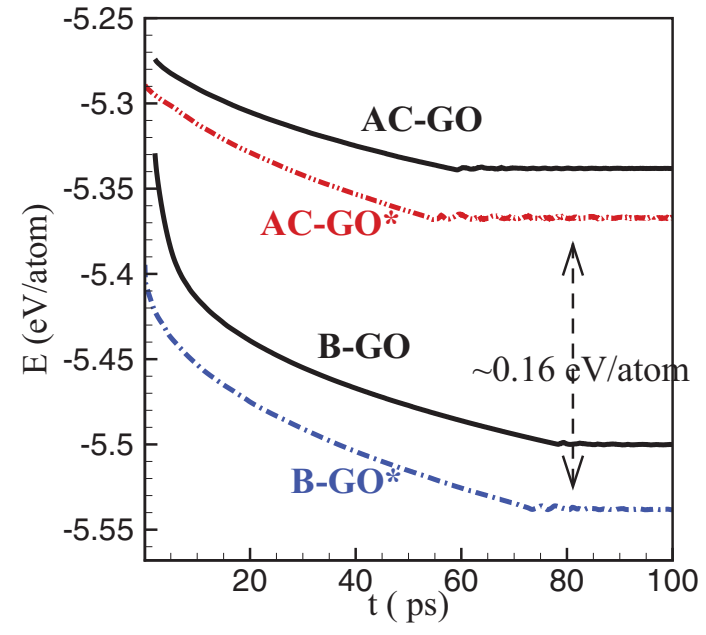

FIG. 9. (Color online) Variation of the total energy with time, for both armchairlike and boatlike graphone.

[Fig. 11(b)], and AC-GFH [Fig 11(c)] after 25 ps of molecular dynamics simulations (red, green, and blue balls are carbon, fluor, and hydrogen, respectively). In all three cases, we used the ReaxFF potential and the simulations were done at $T=$ $10 \mathrm{~K}$, as before. It is clear from Fig. 11 that AC-GH (AC-GFH) has the largest (smallest) curvature due to the fact that when one side is covered by hydrogen and the other side is covered by fluor, the driven force for rolling is partially balanced, but not completely, as the fluor atoms are larger, which leads to a stronger intralayer repulsion at the fluor side, so that the system still tends to roll, albeit with a much smaller curvature.

Before ending this section, it is worthwhile to mention that the spiral deformation was also found for graphene sheets with lower one-sided coverages of $\mathrm{H}$ or $\mathrm{F}$ atoms, e.g., $25 \%$, i.e., $\mathrm{C}_{4} \mathrm{~F}$. The latter system was experimentally realized by Robinson et al. ${ }^{41}$ We performed simulations for both $\mathrm{C}_{4} \mathrm{~F}$ and

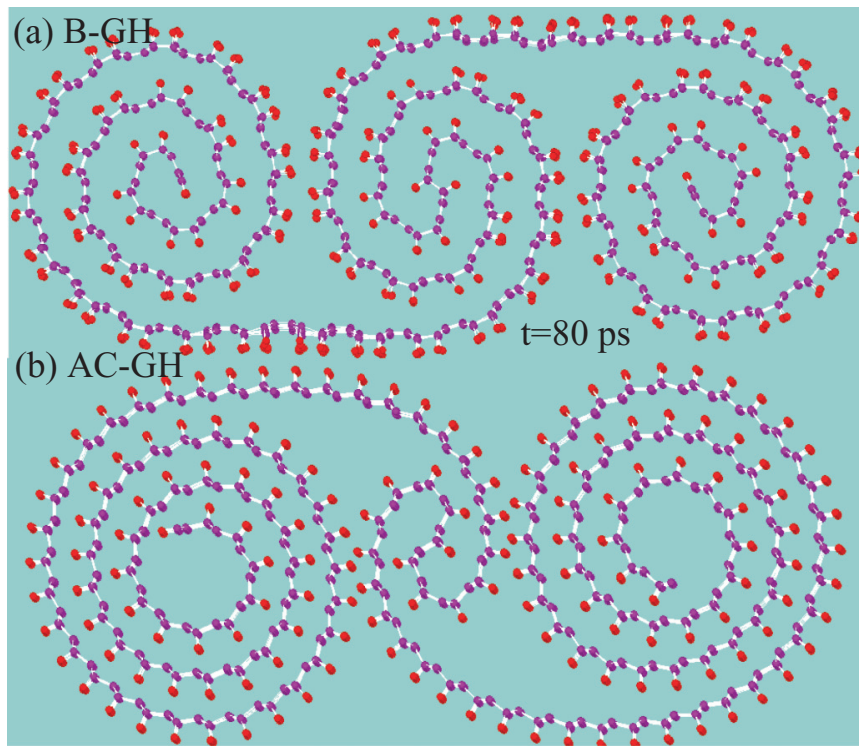

FIG. 10. (Color online) The optimized configuration of (a) boatlike and (b) armchairlike semi-infinite graphone in (a) the A-lattice and (b) the B-lattice carbon sites which were covered by hydrogens from top and bottom, respectively.

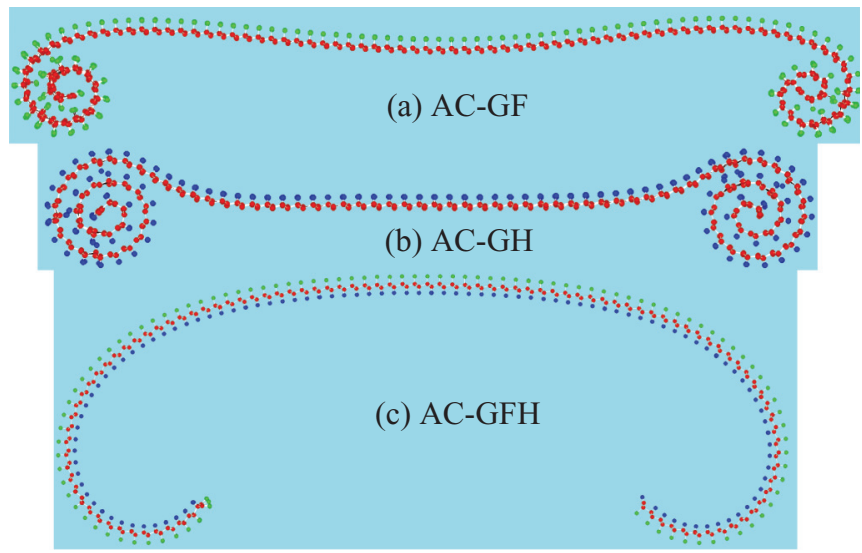

FIG. 11. (Color online) Side view of three different spiral structures: (a) AC-GF, (b) AC-GH, and (c) AC-GFH after 25 ps of relaxation from the starting flat geometry.

$\mathrm{C}_{4} \mathrm{H}$ and found a similar rolling characteristic. Two snapshots of these simulations are shown in Fig. 12. The only difference (compared to GF and GH) is the smaller curvature of the rolled $\mathrm{C}_{4} \mathrm{~F}$ and $\mathrm{C}_{4} \mathrm{H}$ spiral structures compared to those with $50 \%$ coverage.

\section{E. Temperature effects}

These structures have to be tested in terms of their stability against temperature. In order to study the temperature effects on the spiral B-GH, after obtaining it at $10 \mathrm{~K}$ (note that in principle spiral $\mathrm{GH}$ can also be formed at room temperature, but we set $T=10 \mathrm{~K}$ in order to minimize thermal fluctuations and to more rapidly find the ground-state spiral configurations), we raised the temperature up to $1000 \mathrm{~K}$. It is not so surprising in view of the significant gain in binding energy of $0.1 \mathrm{eV} /$ atom $(1200 \mathrm{~K})$ for AC-GH and even more for B-GH (see Fig. 9) that in their rolled states, with respect to the flat configurations, the spiral GHs keep their configuration. Beyond $700 \mathrm{~K}$, the loops start to deform slightly. However, even at $1000 \mathrm{~K}$, the system is not unzipped and the spiral configuration is conserved, ${ }^{39}$ at least within the MD simulation time of $1 \mathrm{~ns}$ used here. For $T>1000 \mathrm{~K}$, the system starts to separate into two different loops ${ }^{39}$ From these simulations, we conclude that spiral GH are rather stable at room temperature, facilitating its possible synthesis in future experiments. Two movies are provided in the Supplemental Material ${ }^{39}$ to show

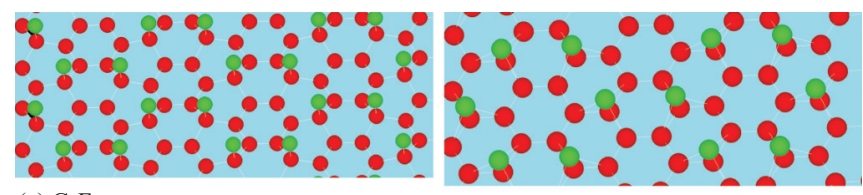

(a) $\mathrm{C}_{4} \mathrm{~F}$ (b) $\mathrm{C}_{4} \mathrm{H}$

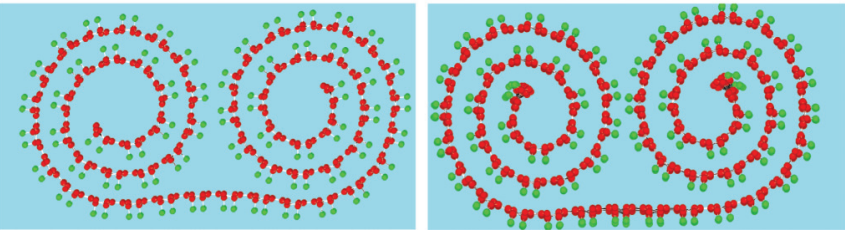

FIG. 12. (Color online) Top view and rolled (a) $\mathrm{C}_{4} \mathrm{~F}$ and (b) $\mathrm{C}_{4} \mathrm{H}$. 
the rolling of B-GH at $T=10 \mathrm{~K}$ and to show the stability of B-GH during the heating process up to $T=1000 \mathrm{~K}$. For GFs, the spiral system is somewhat less stable. Close to $800 \mathrm{~K}$, the GFs start to unroll and, beyond this temperature, they start to be fractured. Notice that the $\mathrm{C}-\mathrm{H}$ bonds (C-F bonds) in GHs (GFs) are stable even after unrolling, thus no hydrogen release was observed when heating up the rolled sheets within our simulation time of $0.1 \mathrm{~ns}$. However, the absence of hydrogen dissociation at high $T$, e.g., above $800 \mathrm{~K}$ where dissociation is observed experimentally, might simply be due to a barrier for the $\mathrm{C}-\mathrm{H}$ bond breaking, and the fact that the time scale of our simulation or, more generally, the typical time scales in MD simulations $(\sim \mathrm{ns})$ is too short to overcome this barrier. In a recent theoretical study, the desorption time for hydrogenated graphene has been determined to be $10^{3} \mathrm{~s}$ using Arrhenius' equation. ${ }^{40,42}$

We also applied external uniaxial compression along the axis of the spiral structures in order to investigate the mechanical stability of these structures, which turned out to resist against the external pressure much better than graphene. The detailed mechanical properties of these spiral hydrocarbons need further investigation.

\section{CONCLUSIONS}

Free boatlike and armchairlike one-sided hydrogenated/ fluorinated graphene (graphone/fluorographene) ribbons/ flakes spontaneously roll up to form spiral configurations, which were shown to be stable at room temperature. The spiral structures exhibit strong mechanical rigidity that prevents them from unrolling. The main driving force behind the spiral formation is the energy relaxation associated with the one-sided, asymmetric orientation of $s p^{3}$ bonding. Further stabilizing factors are the van der Waals attractive interaction between the stacked shells and the ionic interactions between the shells, which appear to be polarized across the shell width. Boatlike hydrogenated/fluorinated graphene yields more stable, spiral systems than armchairlike graphone. The graphene sheet where one side is covered by hydrogen and the other side is covered by fluor is unstable and it also forms a rolled up structure, albeit with a smaller curvature. The highest occupied and lowest unoccupied orbital for spiral GH and GF are localized at opposite ends of the system. The energy gap increases when the system evolves from the flat to the spiral shape.

\section{ACKNOWLEDGMENTS}

We thank A. Sadeghi, M. R. Ejtehadi, and J. Amini for their useful comments. This work is supported by the ESF EuroGRAPHENE project CONGRAN and the Flemish Science Foundation (FWO-Vl). M.N.-A. is supported by a EU-Marie Curie IIF fellowship program Grant No. 299855.
*Corresponding author: neekamal@ srttu.edu

${ }^{1}$ A. K. Geim and K. S. Novoselov, Nature Mater. 6, 183 (2007);

A. H. Castro Neto, F. Guinea, N. M. Peres, K. S. Novoselov, and

A. K. Geim, Rev. Mod. Phys. 81, 109 (2009).

${ }^{2}$ D. Pacile, J. C. Meyer, C. O. Girit, and A. Zettl, Appl. Phys. Lett. 92, 133107 (2008).

${ }^{3}$ P. Vogt, P. DePadova, C. Quaresima, J. Avila, E. Frantzeskakis, M. C. Asensio, A. Resta, B. Ealet, and G. Le Lay, Phys. Rev. Lett. 108, 155501 (2012).

${ }^{4}$ G-H. Lee, Y-J. Yu, C. Lee, C. Dean, K. L. Shepard, P. Kim, and J. Hone, Appl. Phys. Lett. 99, 243114 (2011).

${ }^{5}$ K. S. Novoselov, A. K. Geim, S. V. Morozov, D. Jiang, Y. Zhang, S. V. Dubonos, I. V. Grigorieva, and A. A. Firsov, Science 306, 666 (2004).

${ }^{6}$ M. H. F. Sluiter and Y. Kawazoe, Phys. Rev. B 68, 085410 (2003); J. O. Sofo, A. S. Chaudhari, and G. D. Barber, ibid. 75, 153401 (2007).

${ }^{7}$ R. Nair, W. Ren, R. Jalil, I. Riaz, V. Kravets, L. Britnell, P. Blake, F. Schedin, A. Mayorov, S. Yuan, M. Katsnelson, H. Cheng, W. Strupinski, L. Bulusheva, A. Okotrub, I. Grigorieva, A. Grigorenko, K. Novoselov, and A. K. Geim, Small 6, 2877 (2010).

${ }^{8}$ M. Neek-Amal and F. M. Peeters, Phys. Rev. B 83, 235437 (2011); S. Costamagna, M. Neek-Amal, J. H. Los, and F. M. Peeters, ibid. 86, 041408 (2012).

${ }^{9}$ D. W. Boukhvalov, M. I. Katsnelson, and A. I. Lichtenstein, Phys. Rev. B 77, 035427 (2008).

${ }^{10}$ D. C. Elias, R. R. Nair, T. M. G. Mohiuddin, S. V. Morozov, P. Blake, M. P. Halsall, A. C. Ferrari, D. W. Boukhvalov, M. I. Katsnelson, A. K. Geim, and K. S. Novoselov, Science 323, 610 (2009).
${ }^{11}$ M. Z. S. Flores, P. A. S. Autreto, S. B. Legoas, and D. S. Galvao, Nanotechnology 20, 465704 (2009).

${ }^{12}$ O. Leenaerts, H. Peelaers, A. D. Hernández-Nieves, B. Partoens, and F. M. Peeters, Phys. Rev. B 82, 195436 (2010).

${ }^{13}$ A. D. Hernández-Nieves, B. Partoens, and F. M. Peeters, Phys. Rev. B 82, 165412 (2010).

${ }^{14}$ X. D. Wen, L. Hand, V. Labet, T. Yang, R. Hoffmann, N. W. Ashcroft, A. Oganov, and A. Lyakhov, Proc. Natl. Acad. Sci. 108, 6833 (2011).

${ }^{15}$ L. Hornekaer, Z. Sljivancanin, W. Xu, R. Otero, E. Rauls, I. Stensgaard, E. Laegsgaard, B. Hammer, and F. Besenbacher, Phys. Rev. Lett. 96, 156104 (2006).

${ }^{16}$ F. Dumont, F. Picaud, C. Ramseyer, C. Girardet, Y. Ferro, and A. Allouche, Phys. Rev. B 77, 233401 (2008).

${ }^{17}$ Z. Slijvancanin, R. Balog, and L. Hornekær, Chem. Phys. Lett. 541, 70 (2012)

${ }^{18}$ H. Sahin, C. Ataca, and S. Ciraci, Phys. Rev. B 81, 205417 (2010).

${ }^{19}$ R. Balog, B. Jørgensen, L. Nilsson, M. Andersen, E. Rienks, M. Bianchi, M. Fanetti, E. Lægsgaard, A. Baraldi, S. Lizzit, Z. Sljivancanin, F. Besenbacher, B. Hammer, T. G. Pedersen, P. Hofmann, and L. Hornekr, Nature Mater. 9, 315 (2010).

${ }^{20}$ Duminda K. Samarakoon and Xiao-Qian Wang, ACS Nano 3, 4017 (2009).

${ }^{21}$ J. Zhou, Q. Wang, Q. Sun, X. C. Chen, Y. Kawazoe, and P. Jena, Nano Lett. 9, 3867 (2009).

${ }^{22}$ S. Lebegue, M. Klintenberg, O. Eriksson, and M. I. Katsnelson, Phys. Rev. B 79, 245117 (2009).

${ }^{23}$ Konstantin N. Kudin, Gustavo E. Scuseria, and B. I. Yakobson, Phys. Rev. B 64, 235406 (2001). 
${ }^{24}$ Ji Zang, Minghuang Huang, and Feng Liu, Phys. Rev. Lett. 98, 146102 (2007).

${ }^{25}$ V. V. Ivanovskaya, P. Wagner, A. Zobelli, I. Suarez-Martinez, A. Yaya, and C. P. Ewels, Carbon Nano. 25, 75 (2012).

${ }^{26}$ D. Yu and F. Liu, Nano Lett. 7, 3046 (2007).

${ }^{27}$ O. O. Kit, T. Tallinen, L. Mahadevan, J. Timonen, and P. Koskinen, Phys. Rev. B 85, 085428 (2012).

${ }^{28}$ D. V. Kosynkin, A. L. Higginbotham, A. Sinitskii, J. R. Lomeda, A. Dimiev, B. K. Prince, and J. M. Tour, Nature (London) 458, 872 (2009).

${ }^{29}$ L. Jiao, L. Zhang, X. Wang, G. Diankov, and H. Dai, Nature (London) 458, 877 (2009).

${ }^{30}$ Juan J. Vilatela and Dominik Eder, ChemSusChem 5, 456 (2012).

${ }^{31}$ F. Alvarez-Ramírez, J. A. Toledo-Antonio, C. Angeles-Chavez, J. H. Guerrero-Abreo, and E. Lopez-Salinas, J. Phys. Chem. C 115, 11442 (2011).

${ }^{32}$ G. Shen, B. Liang, X. Wang, P-C. Chen, and C. Zhou, ACS Nano 5, 2155 (2011).

${ }^{33}$ K. Fukui, T. Yonezawa, and H. Shingu, J. Chem. Phys. 20, 722 (1952).
${ }^{34}$ M. J. Frisch et al., computer code GAUSSIAN (1998).

${ }^{35}$ S. J. Plimpton, J. Comp. Phys. 117, 1 (1995); http://lammps.sandia.gov.

${ }^{36}$ S. J. Stuart, A. B. Tutein, and J. A. Harrison, J. Chem. Phys. 112, 6472 (2000).

${ }^{37}$ A. C. T. van Duin, S. Dasgupta, F. Lorant, and W. A. Goddard, J. Phys. Chem. A 105, 9396 (2001).

${ }^{38}$ D. W. Brenner, O. A. Shenderova, J. A. Harrison, S. J. Stuart, B. Ni, and S. B. Sinnot, J. Phys.: Condens. Matter 14, 783 (2002).

${ }^{39}$ See Supplemental Material at http://link.aps.org/supplemental/ 10.1103/PhysRevB.87.075448 for five movies of the rolled GH and GF, and the temperature effect and the formation of CNT.

${ }^{40}$ M. Klintenberg, S. Lebégue, M. I. Katsnelson, and O. Eriksson, Phys. Rev. B 81, 085433 (2010).

${ }^{41}$ J. T. Robinson, J. S. Burgess, C. E. Junkermeier, S. C. Badescu, T. L. Reinecke, F. K. Perkins, M. K. Zalalutdniov, J. W. Baldwin, J. C. Culbertson, P. E. Sheehan, and E. S. Snow, Nano Lett. 8, 3001 (2010).

${ }^{42}$ L. A. Openov and A. I. Podlivaev, Tech. Phys. Lett. 36, 69 (2010). 